\title{
Overexpression of human telomerase reverse transcriptase promotes the motility and invasiveness of HepG2 cells in vitro
}

\author{
PENG-CHENG CHEN, JI-RUN PENG, LEI HUANG, WEN-XIA LI, WEN-ZHEN WANG, \\ ZHU-QING-QING CUI, HUI HAN, LEI GONG, DA-PENG XIANG, SHI-SHI QIAO, \\ XIN YU, YU-HUA WEI, LI-PING MA, NA LI, JI-YE ZHU and XI-SHENG LENG
}

Peking University People's Hospital, Beijing 100044, P.R. China

Received April 2, 2013; Accepted May 2, 2013

DOI: $10.3892 / o r .2013 .2563$

\begin{abstract}
Recent studies have indicated that telomerase activity promotes cancer invasion and metastasis, but the underlying mechanism remains unclear. Several studies have shown that expression of exogenous human telomerase reverse transcriptase (hTERT) can promote motility and invasiveness among telomerase-negative tumor cells, and inhibition of endogenous telomerase activity can reduce invasiveness in tumor cells. However, whether overexpression of hTERT can further enhance the motility and invasiveness of telomerase-positive tumor cells has yet to be determined. In the present study, we showed that stable overexpression of hTERT can increase telomerase activity and telomere length, which significantly promotes the invasive and metastatic potential of telomerase-positive HepG2 cells but does not affect cell proliferation. Further analysis suggested that enhanced invasiveness and metastasis may act through corresponding upregulation of mRNA and protein expression of matrix metalloproteinase 9 (MMP9) and Ras homolog gene family member C (RhoC). Our study indicated that exogenous expression of hTERT may promote invasiveness and metastasis through upregulation of MMP9 and RhoC.
\end{abstract}

\section{Introduction}

Human telomerase, a ribonucleoprotein enzyme complex, is composed of catalytic component human telomerase reverse transcriptase (hTERT), human telomerase RNA component (RNA template hTERC) and human telomerase associated protein 1 (hTEP1) (1-3). hTERT can catalyze the synthesis of the repeating sequence (TTAGGG)n and maintain telomere length at chromosomal ends using hTERC as a template (4). It counteracts telomere shortening due to each round of cell

Correspondence to: Professor Xi-Sheng Leng, Department of Hepatobiliary Surgery, Peking University People's Hospital, 11 Xizhimen South Street, Beijing 100044, P.R. China

E-mail: lengxs2003@yahoo.com.cn

Key words: telomerase, human telomerase reverse transcriptase, retroviral vector, HepG2, motility, invasiveness division and therefore prevents senescence and cellular aging. In line with these conclusions, telomerase activity has been detected in $80-90 \%$ of human cancer cases, but it is rare in normal somatic cells. Exceptions include stem cells, reproductive cells and activated lymphocytes. (5-11). It has been shown in numerous studies that activation of telomerase is strongly correlated with tumorigenesis and metastasis; therefore, it may serve as an indicator of prognosis (12-21).

Previous studies have shown that the majority of hepatocellular carcinomas (HCCs) exhibit telomerase activity and that the level of telomerase activity is related to upregulated hTERT expression (22). Early recurrence after hepatectomy is one of the most important factors affecting the prognosis of patients with HCC. Quantitative analyses of telomerase activity suggested that $\mathrm{HCC}$ resection patients positive for telomerase activity in non-cancerous liver tissue have a higher rate of recurrence. The relative telomerase activity (RTA) of early recurrent patients is significantly higher than in patients who do not experience recurrence (23). Peripheral blood telomerase activity can be used as a molecular marker for the detection of circulating hepatoma cells in the blood of HCC patients; it also indicates hematogenous micrometastasis (24). Previous studies have consistently shown that telomerase activity may serve as an independent predictor of recurrence after HCC resection (25). These observations led to the hypothesis that telomerase activity may be correlated to invasiveness and metastasis of cancer. To support this hypothesis, recent research data was collected and showed that telomerase can promote the invasion and metastasis of telomerase-negative tumor cells (26-28). By contrast, tumor cell growth, proliferation, invasion and metastasis can be reduced through inhibition of telomerase activity of tumor cells by various methods $(29,30)$. However, to date, few research studies have focused on the mechanisms underlying this process. The issue of whether the motility and invasiveness of telomerase-positive tumor cells can be further promoted by overexpression of hTERT has yet to be addressed.

In the present study, we observed enhanced motility and invasive capacity in telomerase-positive human hepatoma cell line (HepG2) cells through exogenous expression of hTERT gene using a retroviral vector. Our data suggested that changes in motility and invasive capacity may be caused by upregulation of the metastasis-related genes, matrix metalloproteinase 9 (MMP9) and Ras homolog gene family member C (RhoC). 


\section{Materials and methods}

Cell lines and retroviral vectors. Human hepatoma cell line (HepG2) (ATCC, Manassas, VA, USA) and GP2-293 packaging cell line (Clontech Laboratories, Mountain View, CA, USA) were cultured in Dulbecco's modified Eagle's medium (DMEM; Invitrogen Life Technologies, Carlsbad, CA, USA) supplemented with $10 \%$ fetal bovine serum (FBS; Gibco-BRL, Carlsbad, CA, USA) at $37^{\circ} \mathrm{C}$ in a humidified incubator containing $5 \% \mathrm{CO}_{2}$. The retroviral expression vector $x \operatorname{lox}(\mathrm{gfp})$ TERT containing coding sequences for hTERT and green fluorescent protein (GFP) was provided by Dr David Ott (Frederick National Laboratory for Cancer Research, Frederick, MD, USA). The control vector xlox-GFP was constructed on the basis of xlox(gfp)TERT in our laboratory. Briefly, the portion of hTERT in xlox(gfp)TERT was removed using EcoRI followed by religation using T4 DNA ligase.

Cell transduction. Retroviral vector stocks were produced by cotransfecting the plasmid encoding VSV-G protein and xlox(gfp)TERT/xlox-GFP vector into the packaging cell line GP2-293 and harvesting virus-containing culture medium $48 \mathrm{~h}$ after transfection. These actions were performed as described in the Retroviral Gene Transfer and Expression user manual and instructions from VigoFect (transfection reagent; Vigorous Biotechnology, Beijing, China). A 2:1 ratio of retroviral vector/ VSV-G DNA mixture was used in these experiments. The resulting vector stocks (Virus-hTERT and Virus-GFP) were then used to infect HepG2 cells in the presence of polybrene $(8 \mu \mathrm{g} / \mathrm{ml})$. Two groups of transduced cells were identified and sorted $72 \mathrm{~h}$ after transduction using flow cytometry for GFP expression, termed hTERT/HepG2 and GFP/HepG2. Both untransduced HepG2 and GFP/HepG2 were used as the controls and cultured at the same time as the experimental groups.

Real-time PCR for hTERT mRNA. Total RNA was isolated from cells using TRIzol reagent (Invitrogen Life Technologies) according to the manufacturer's instructions. RNA was then converted to cDNA using oligo(dT) primers and SuperScript III Reverse Transcriptase (Invitrogen Life Technologies). Real-time PCR analysis was performed using the SYBR ${ }^{\circledR}$ Green Real-time PCR Master mix (Toyobo, Japan) and Bio-Rad (Hercules, CA, USA) MiniOpticon real-time PCR system. Primers for human $\beta$-actin and hTERT were designed with Primer Express Software Version 3.0 and synthesized by Sangon Biotech Co. (Shanghai, China), and were: $\beta$-actin sense, 5'-TGG ACTTCGAGCAAGAGATG-3' and antisense, 5'-GAAGGAAGGCTGGAAGAGTG-3' (137 bp); hTERT sense, 5'-TGTCAAGGTGGATGTGACGGGC-3' and antisense, 5'-GGCATACC GACGCACGCAGT-3' (112 bp).

hTERT protein detection. Expression of the hTERT protein was detected using western blot analysis. Total proteins were isolated from cells of each group. Forty micrograms of protein were separated using $10 \%$ sodium dodecyl sulfate-polyacrylamide gel electrophoresis (SDS-PAGE) and then transferred onto a nitrocellulose membrane (Bio-Rad). The membranes were first incubated with anti-hTERT mAb overnight at $4^{\circ} \mathrm{C}$ and then with HRP-labeled secondary antibody (Zhong-Shan Golden Bridge BioTechnology, China) for $1 \mathrm{~h}$ at RT. The membranes were washed again, treated with chemiluminescence reagent (Amersham Pharmacia Biotech, Amersham, UK), exposed to autoradiography film (Kodak) and developed.

TRAP assay for telomerase activity. Telomerase activity of cells was detected using the telomeric repeat amplification protocol (TRAP) assay with a TRAP kit (Tiandz, Beijing, China). The procedure was performed according to the manufacturer's instructions. Briefly, the cell pellet was resuspended in $200 \mu \mathrm{l}$ of $1 \mathrm{X}$ lysis buffer $/ 10^{6}$ cells, incubated on ice for $30 \mathrm{~min}$ and then centrifuged at $12,000 \mathrm{rpm}$ for $20 \mathrm{~min}$ at $4^{\circ} \mathrm{C}$. The supernatant was collected and the protein concentration was determined using standard procedures (BCA protein assay). The reaction system containing $25 \mu 1$ TRAP reagent was incubated for $30 \mathrm{~min}$ at $30^{\circ} \mathrm{C}$ and then for $3 \mathrm{~min}$ at $93^{\circ} \mathrm{C}$, and $1.5 \mu 1$ TRAP primer mixture and $0.5 \mu 1$ of Taq polymerase were added to the PCR tube followed by 30 cycles of $94^{\circ} \mathrm{C}$ for $30 \mathrm{sec}, 59^{\circ} \mathrm{C}$ for $30 \mathrm{sec}$ and $72^{\circ} \mathrm{C}$ for $60 \mathrm{sec}$. PCR product $(25 \mu \mathrm{l})$ was loaded onto a $10 \%$ non-denaturing PAGE in $0.5 \mathrm{X}$ TBE buffer. Following electrophoresis, the gel was stained using a Rapid Silver Stain kit for Nucleic Acid (Tiandz). Lysis buffer (provided in the TRAP kit) was used as a negative control.

FISH assay for telomere. hTERT/HepG2 and GFP/HepG2 cells $\sim 10$ PDs after transduction and untransduced cells were cultured in a 6-well plate to the logarithmic phase. Then, colcemid was added and the cells were incubated for $1 \mathrm{~h}$. Cells were then treated with hypotonic $\mathrm{KCl}$ for $60 \mathrm{~min}$ at $37^{\circ} \mathrm{C}$ and fixed in methanol/acetic-acid. Fluorescence in situ hybridization (FISH) was performed on metaphase chromosomes with Cy-3 labeled (CCCTAA) $)_{3}$ PNA probe (Panagen, South Korea) according to the manufacturer's instructions. Digital images were captured for 4,6 diamidino-2-phenylindole (DAPI) and Cy-3. The subsequent quantitative analysis of telomere fluorescence was detected using Leica QWin Pro. version 2.6 (Germany).

In vitro motility assay and invasiveness assay. Cell motility was analyzed using a wound healing assay. Cells $\left(5 \times 10^{5}\right.$ cells/well) were seeded into a 6-well plate. A scratch wound in the monolayer was created using a sterile $200 \mu 1$ pipette tip following incubation for $24 \mathrm{~h}$. The detached cells were gently washed with PBS. The cells were incubated with new medium containing $1 \%$ FBS and then the distances between the wounds were measured by microscope at 0 and $48 \mathrm{~h}$. These distances were measured using ImageJ. Cell motility was evaluated using the following formula: Cell motility $=\left(\right.$ distance $_{48 \mathrm{~h}}-$ distance $\left.{ }_{0 \mathrm{~h}}\right) /$ distance $_{0 \mathrm{~h}}$. For invasiveness assays, cells $\left(5 \times 10^{4}\right.$ cells/well) were suspended in DMEM with no FBS and seeded onto the inner compartment of Matrigel-coated 24-well chambers with a filter membrane containing $8-\mu \mathrm{m}$ pores (Corning Incorporated, Corning, NY, USA). The outer chamber contained the same medium with $10 \% \mathrm{FBS}$. Following incubation for $24 \mathrm{~h}$ at $37^{\circ} \mathrm{C}$, cells that did not migrate through the pores were removed by gentle scraping of the membrane with a cotton swab. Cells that transversed the membrane were fixed in $70 \%$ ethanol and stained with $0.1 \%$ crystal violet. The number of cells that invaded the undersurface of the membrane was determined using 5 randomly selected microscopic fields per sample. Data represent the average of 3 wells. 

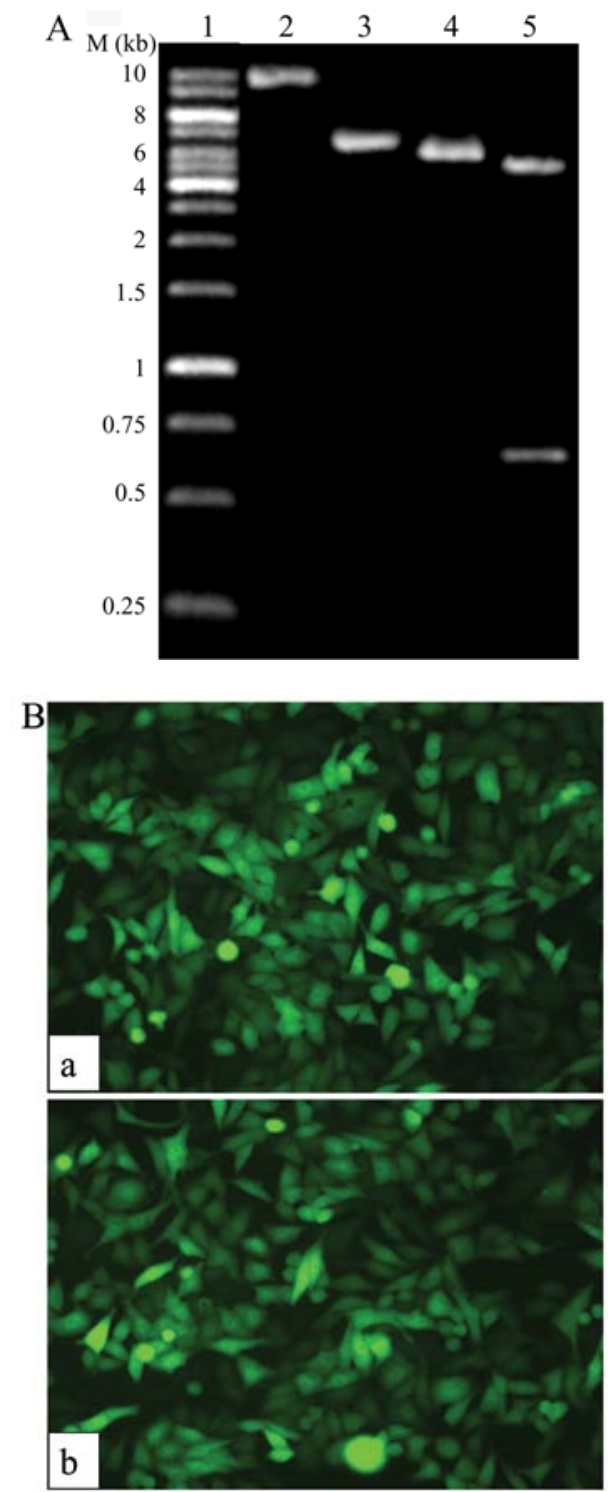

Figure 1. Control vector and transduced cells. (A) Lane 1, marker (10 kb); lane 2, xlox(gfp)TERT vector; lane 3, control (xlox-GFP) vector; lane 4 xlox-GFP was digested with EcoRI, and no fragment was observed after electrophoresis. Lane 5, xlox-GFP was digested with NotI and GFP fragment $(0.72 \mathrm{~kb})$ was observed after electrophoresis. (B) Two groups of transduced cells were sorted for GFP expression using flow cytometry, (a) hTERT/ HepG2 and (b) GFP/HepG2.

Cell growth curves. Cell proliferation was determined using Cell Counting Kit-8 (CCK-8, Dojindo, Japan) according to the manufacturer's instructions. One hundred microliters of cell suspension $(2,000$ cells/well) were seeded into the 96 -well plate and incubated for $24 \mathrm{~h}\left(37^{\circ} \mathrm{C}, 5 \% \mathrm{CO}_{2}\right)$. CCK-8 solution (10 $\mu \mathrm{l})$ was added to each well and the cultures were incubated at $37^{\circ} \mathrm{C}$ for $90 \mathrm{~min}$. Absorbance at $450 \mathrm{~nm}$ was measured using an automatic microplate reader (Tecan Sunrise, Switzerland). The results were plotted as means \pm SD of 3 separate experiments having 6 determinations per experiment for each experimental condition.

Real-time PCR for MMP9 and RhoC $m R N A$. The expression levels of mRNA for MMP9 and RhoC were analyzed using real-time PCR as previously described. Primers for MMP9
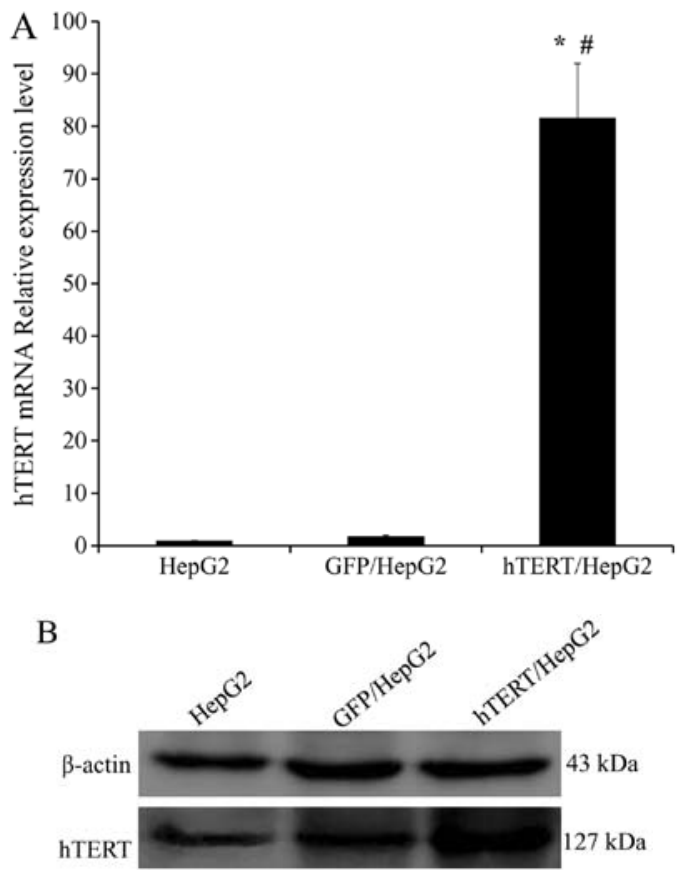

Figure 2. Level of hTERT mRNA and protein expression of HepG2, GFP/HepG2 and hTERT/HepG2. (A) Real-time PCR for hTERT. Data are means \pm SD of 3 independent experiments $\left({ }^{*} \mathrm{P}<0.05\right.$, relative to HepG 2 mean values; ${ }^{\text {P }}<0.05$, relative to GFP/HepG2 mean values, ANOVA). (B) Western blot analysis of hTERT protein. The data are representative of 3 independent experiments.

and RhoC were designed with Primer Express Software version 3.0 and synthesized by Sangon Biotech Co., and were: MMP9 sense, 5'-GGCGGTGATTGACGACGCCT-3' and antisense, 5'-CCGTGCTCCGCGACACCAAA-3' (126 bp); RhoC sense, 5'-CGGAGCGGAAGCCCCACCAT-3' and antisense, 5'-AGGGACGTAGACCTCCGGAAACT-3' (126 bp).

MMP9 and RhoC protein detection. The expression levels of proteins for MMP9 and RhoC were detected using western blot analysis, as previously described.

Statistical analysis. All experiments were performed in triplicate and data are presented as means \pm SD of 3 separate determinations. Statistical analysis was performed using one-way analysis of variance (ANOVA). $\mathrm{P}<0.05$ was considered to indicate statistically significant differences. All statistical analyses were performed using SPSS 16.0 software.

\section{Results}

Control vector construction and cell transduction. The control vector xlox-GFP was constructed on the basis of $x \operatorname{lox}(\mathrm{gfp})$ TERT by removing part of the hTERT in xlox(gfp)TERT with EcoRI and linking it using T4 DNA ligase. The vector was confirmed by digestion with EcoRI and NotI (Fig. 1A). Virus-hTERT and Virus-GFP were produced and used to infect HepG2 cells. Two groups of transduced cells were identified and sorted $72 \mathrm{~h}$ after transduction using flow cytometry for GFP expression, hTERT/HepG2 and GFP/HepG2 (Fig. 1B). The following experiments were performed using hTERT/ HepG2, GFP/HepG2 and HepG2 cells. 


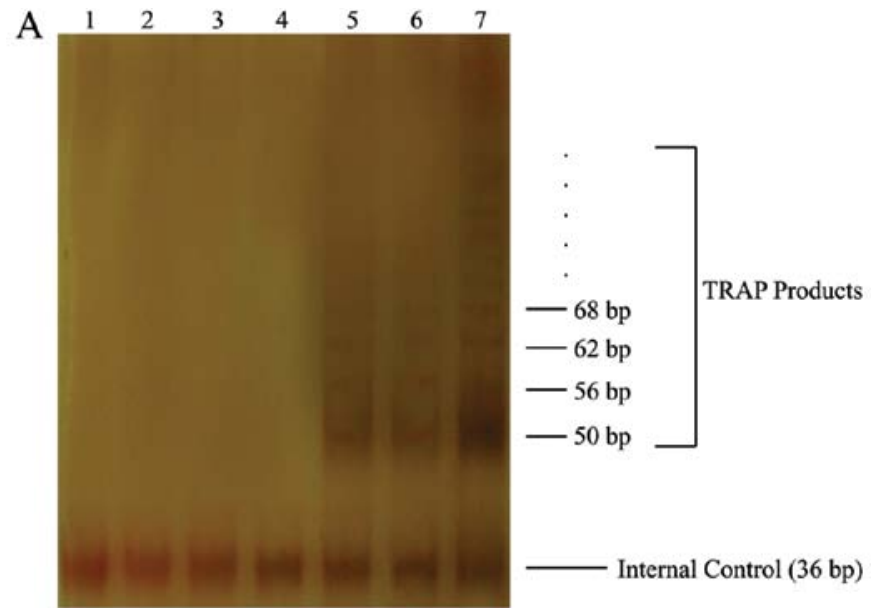

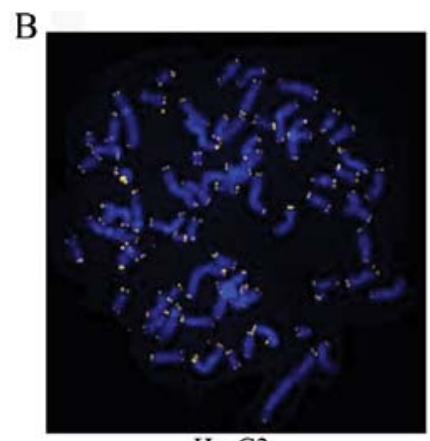

HepG2

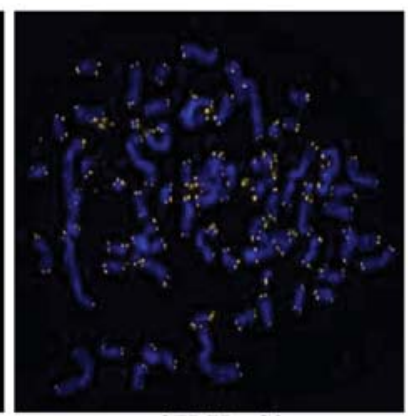

GFP/HepG2

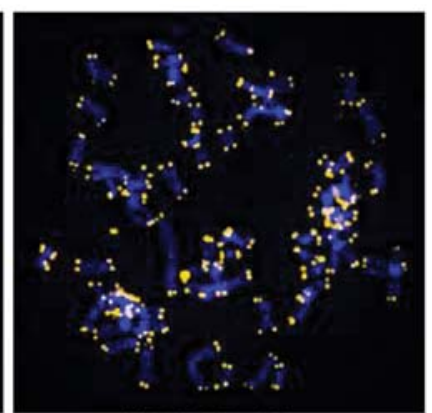

hTERT/HepG2

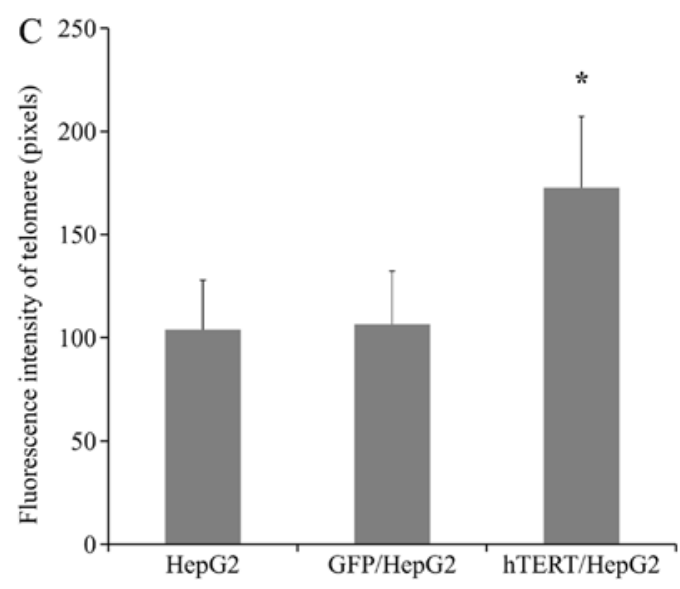

Figure 3. Telomerase activity and telomere fluorescence intensity assay. (A) Telomerase activity (TRAP assay). Lane 1, negative control (1X TRAP lysis buffer); lanes 2-4, heat-treated control cell extracts (HepG2, GFP/HepG2, hTERT/HepG2); lanes 5-7, cell extracts (HepG2, GFP/HepG2, hTERT/HepG2). (B) FISH assay of HepG2, GFP/HepG2, hTERT/HepG2. (C) Quantitative analysis of telomere fluorescence intensity. Data are means $\pm \mathrm{SD}$ ("P $<0.05$, compared with HepG2 mean values; ${ }^{*} \mathrm{P}<0.05$, relative to $\mathrm{GFP} / \mathrm{HepG} 2$ mean values, ANOVA).

Overexpression of $h T E R T$ mRNAand protein in hTERT/HepG2. The expression of hTERT in all 3 groups of cells was examined using real-time PCR. $\beta$-actin served as a loading control for both mRNA and protein analyses. We found hTERT mRNA expression to be markedly higher in hTERT/HepG2 cells (up to 80-fold) than in HepG2 and GFP/HepG2 control cells (Fig. 2A). Elevated hTERT protein levels were also detected in hTERT/HepG2 cells relative to HepG2 and GFP/HepG2 cells (Fig. 2B). There was no significant difference between HepG2 and GFP/HepG2 in either mRNA or protein expression. The relative expression levels of hTERT mRNA in HepG2, GFP/HepG2 and hTERT/HepG2 were 1.00, 1.76 and 81.65 (Fig. 2A). We used this information to generate a stable cell line with high hTERT expression. This line was used for subsequent studies.

hTERT overexpression enhances telomerase activity and telomere length in HepG2 cells. To further examine the effect of hTERT overexpression, TRAP assay was performed to assess telomerase activity in HepG2, GFP/HepG2 and hTERT/HepG2. Our results showed telomerase activity in the hTERT/HepG2 cells to be significantly more pronounced than in the 2 control cells (Fig. 3A). The average length of telomere repeats (indicated by the intensity of telomere fluorescence) at chromosome ends in HepG2, GFP/HepG2 and hTERT/HepG2 was determined using semiquantitative FISH. As in our exper- 

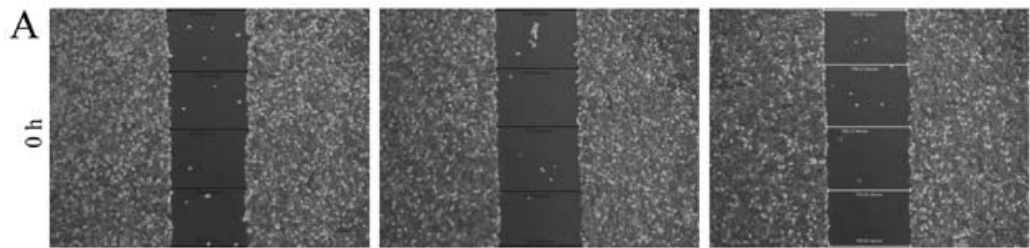

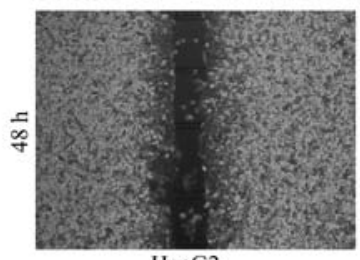

HepG2

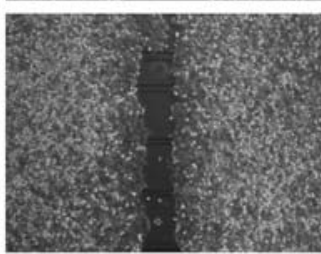

GFP/HepG2

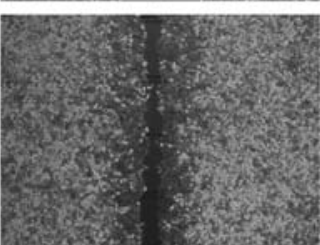

hTERT/HepG2
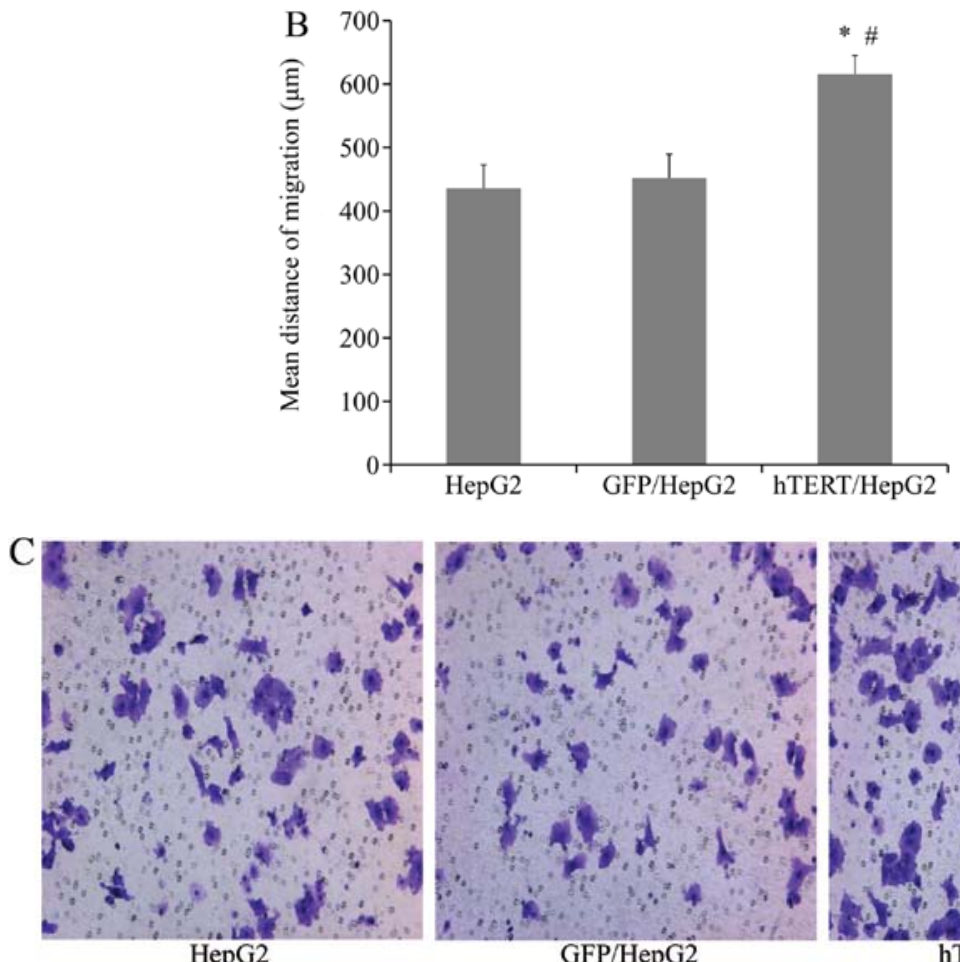

GFP/HepG2
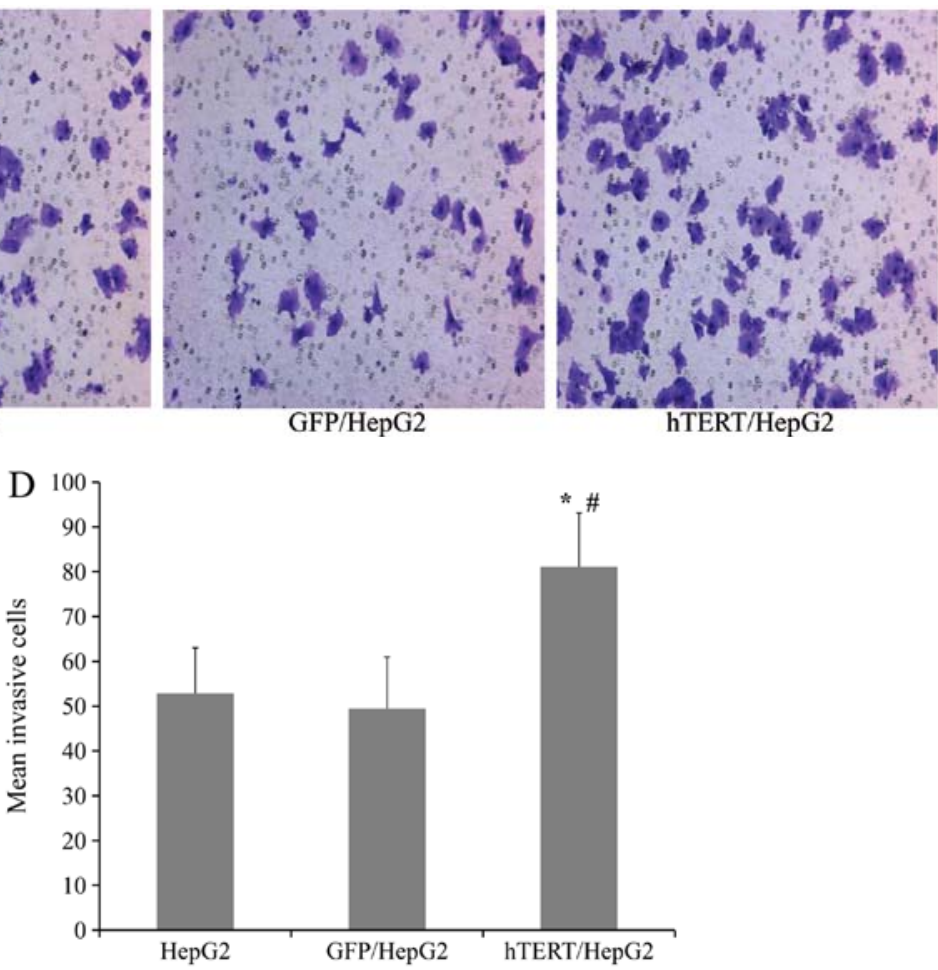

Figure 4. Effects of hTERT transduction on cell migration and invasion in HepG2 cells. (A) Images of wounded monolayers of 3 groups of cells were captured at 0 and $48 \mathrm{~h}$ after incubation (x40). (B) The mean distance of migration of each group of cells was measured and compared. (C) Representative cell Matrigel-coated invasion assay for each group and images of invaded cells at $48 \mathrm{~h}$. (D) Quantitative evaluation of cell invasion assay. (* $\mathrm{P}<0.05$, relative to HepG2 mean values; ${ }^{\mathrm{P}}<0.05$, relative to GFP/HepG2 mean values, ANOVA).

iments on elevated telomerase activity, telomere length, here indicated by fluorescence intensity, was significantly longer in hTERT/HepG2 cells than in HepG2 and GFP/HepG2 control cells (Fig. 3B).
hTERT overexpression leads to increased motility and invasiveness of HepG2 cells. Previous studies conducted using tissue samples from certain cancer patients indicated that levels of high telomerase expression and activity are corre- 


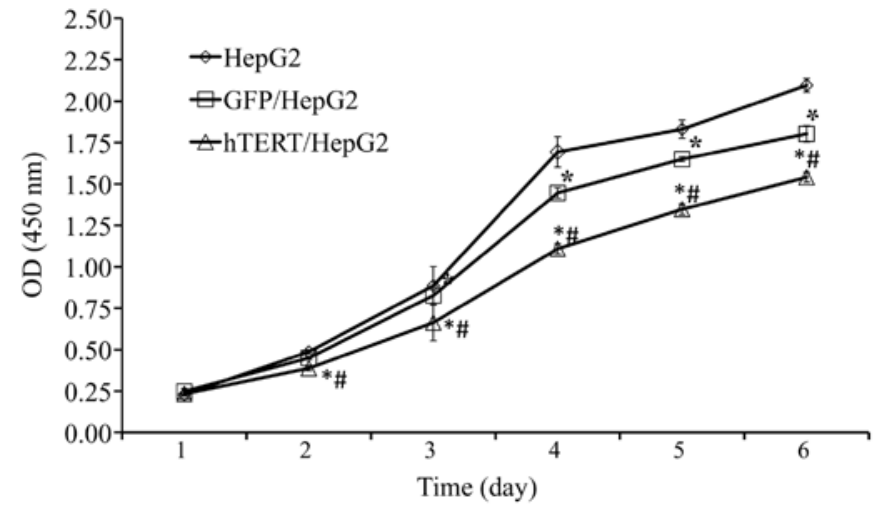

Figure 5. Cell growth curves. Cell proliferation speed of hTERT/HepG2 was significantly inhibited compared with that of HepG2 and GFP/HepG2. ( $\mathrm{P}<0.05$, relative to HepG2 mean values; ${ }^{\prime \prime} \mathrm{P}<0.05$, relative to $\mathrm{GFP} / \mathrm{HepG} 2$ mean values, ANOVA).

lated with increased motility and invasiveness, suggesting that telomerase expression plays a critical role in tumor progression. To assess the motility and invasiveness of infected hTERT/HepG2 cells, we carried out wound healing assay and invasive experiment using Matrigel-coated transwell chambers. As in previous studies, overexpression of hTERT in HepG2 cells led to increased cell motility and invasiveness relative to 2 sets of control cells (Fig. 4). Taken together, our data suggested that increased telomerase expression enhanced cell motility and invasiveness in vitro and facilitated tumor progression towards metastasis stage in vivo.

Overexpression in telomerase-positive HepG2 slows down cell proliferation. It has been suggested that telomerase expression may promote cell proliferation and maintain homeostasis in adult tissue. To determine whether this is the case in HCCs overexpressing hTERT, a CCK- 8 assay was performed to assess the cell proliferation rate. The speed of cell proliferation among hTERT/HepG2 was significantly slower than among GFP/HepG2 and HepG2 control cells (Fig. 5). However, further studies are required to fully clarify this.

Upregulation of MMP9 and RhoC may be responsible for enhanced motility and invasiveness in HepG2 when hTERT expression is forced. In order to determine the molecular mechanism by which hTERT promotes the motility and invasiveness of HepG2 cells, we assessed the mRNA and protein levels of 2 important molecules involved in cell migration and invasion. We observed marked upregulation of RhoC and MMP9 on both the mRNA and protein levels in hTERT/HepG2 (Fig. 6A and B). Our findings suggested that enhanced cell motility and invasiveness induced by high levels of hTERT in HepG2 cells may have been caused by upregulation of MMP9 and RhoC expression.

\section{Discussion}

Aggressiveness is a significant biological characteristic of malignant tumor cells and a leading cause of mortality in patients with cancer. Several studies have demonstrated that telomerase plays an important role in elongating telomeres and
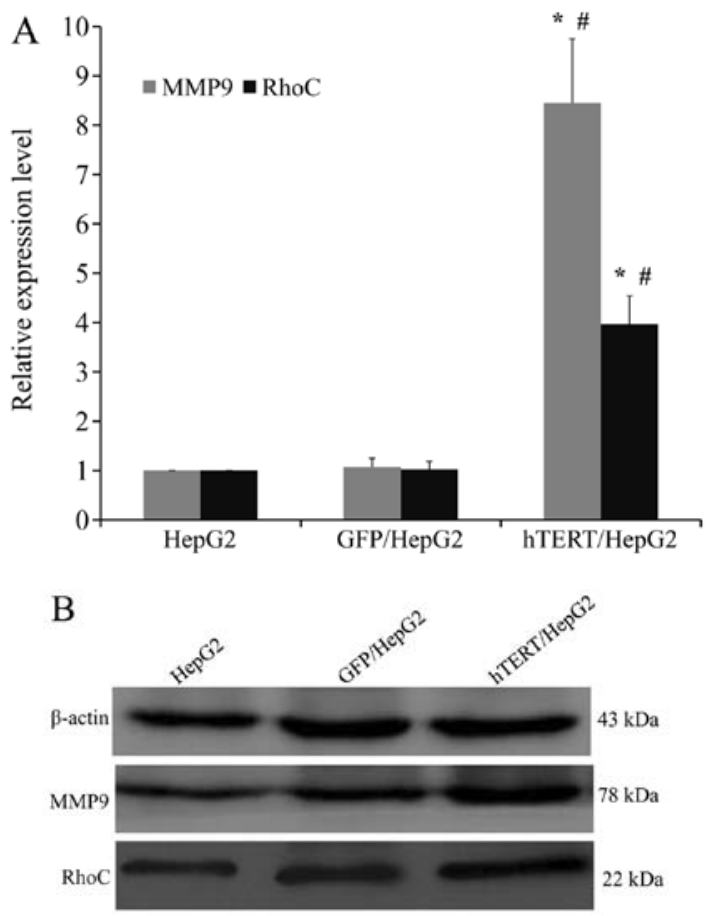

Figure 6. Level of MMP9 and RhoC mRNA and protein expression in HepG2, GFP/HepG2 and hTERT/HepG2. (A) Real-time PCR for MMP9 and RhoC. Data are means \pm SD of 3 independent experiments ( ${ }^{\mathrm{P}}<0.05$, relative to HepG2 mean values; ${ }^{~} \mathrm{P}<0.05$, relative to $\mathrm{GFP} / \mathrm{HepG} 2$ mean values, ANOVA). (B) Western blot analysis for MMP9 and RhoC proteins. The data are representative of 3 independent experiments.

maintaining the infinite proliferation ability of telomerasepositive tumor cells. Clinical findings have indicated that pronounced telomerase activity may be a prognostic marker of various human malignant tumors, including HCC (23). Sato et al (31) found relative telomerase levels to be closely correlated with both motility and invasiveness in 13 pancreatic carcinoma cell lines and concluded that the magnitude of telomerase activation may reflect the potential for aggressive behavior within cancer cells. Previous experimental studies regarding the link between telomerase and tumor aggressiveness have focused on the following two phenomena; first, the transfection or transduction of hTERT can promote the motility and invasiveness of telomerase-negative tumor cells. The invasive and metastatic potential of $\mathrm{Ras}^{\mathrm{G} 12 \mathrm{~V}} / \mathrm{SV} 40$ Large $\mathrm{T}$ antigen-transduced bovine cells can be restored by transduction of hTERT (26). The proliferation, adhesion and invasion of telomerase-negative osteosarcoma cell line U2OS was found to be significantly promoted by hTERT transfection (28). Second, cell proliferation, migration and invasion of telomerase-positive tumor cells can be inhibited by inhibition of either hTERT expression or telomerase activity. Anti-TER ribozyme-mediated suppression of mouse telomerase RNA reduced telomerase RNA expression, telomerase activity and telomere length, which significantly reduced the invasiveness and metastatic potential of melanoma cells (32). Knockdown of hTERT siRNA and concurrent treatment with IFN- $\gamma$ effectively inhibited cell proliferation, migration, and invasion in glioblastoma cells (30). However, to our knowledge, there are no experimental studies on the link between hTERT overexpression and aggressiveness in telomerase-positive tumor cells. 
In the present study, we found that hTERT overexpression can also significantly promote the motility and invasive potential of cancer cells, at least in telomerase-positive HepG2 cell lines. This provides further evidence of the relationship between telomerase activity and metastatic potential of tumor cells.

Tumor cell invasion and metastasis are multi-step, complex processes. They involve proteolytic degradation of basement membranes and extracellular matrix (ECM), cell adhesion, migration and angiogenesis. Both MMPs and RhoC have been shown to play important roles in some of these processes. MMPs are a family of zinc-dependent endoproteinases responsible for degradation of the components of basement membranes and ECM. Overexpression of MMPs has been observed in most malignant tumors. MMP9 expression has been found to be strongly correlated to metastasis of HCC $(33,34)$. Analysis of cell phenotypes expressing dominant-negative Rho or RhoC indicates that RhoC is important in tumor cell invasion (35). RhoC plays a critical role in the movement of tumor cells through regulation of actin cytoskeleton $(36,37)$. RhoC has been shown to be upregulated in various types of tumors, including HCC (38-42). Studies have indicated that RhoC significantly promotes metastasis by augmenting motility and invasion of tumor cells via activation of MMP2 and MMP9 $(43,44)$. In this study, we chose these two key molecules for invasion of tumor cells for our preliminary investigation of the molecular mechanisms by which hTERT promotes motility and invasiveness. We found expression levels of MMP9 and RhoC to be markedly upregulated in hTERT/HepG2 cells at both the mRNA and protein levels. We speculate that upregulation of RhoC and MMP9 may be one of the mechanisms through which hTERT enhances the motility and invasiveness of tumor cells. In this process, there may be complicated regulating networks that control tumor aggressiveness. However, the details of these mechanisms require further study in subsequent experiments.

hTERT is generally considered to be closely related to tumor cell growth and proliferation (45) and it is believed to promote cell growth and proliferation (46-48). However, the few studies that have been performed have shown no significant association between telomerase activity and proliferative index in various tumor tissues $(14,15,31,49)$. Presumably, malignancy and poor outcome among cancer patients may not necessarily be strictly correlated with tumor size or proliferation rate. Other characteristics, such as the degree of differentiation, may be more suitable indicators of malignancy and poor outcome in these patients. In the current study, we showed that cell proliferation was not increased by overexpression of hTERT. Our results also show that cell proliferation is slower in the two transduced groups than in untransduced cells. One possible reason for this may be that forced expression of increased amounts of TERT may cause telomerase-positive cells to acquire some properties and capacities, such as those involving de-differentiation, motility, and invasiveness, but not an increased growth rate. Whatever the reason, the motility and invasiveness of tumor cells were found to have been significantly promoted by overexpression of hTERT, and these were considered indicators of enhanced metastatic potential. In this sense, our results support previous observations that hTERT and telomerase play important roles in the promotion of motility and invasiveness in tumor cells.
The overexpression of hTERT was found to increase the motility and invasiveness of telomerase-positive HepG2 cells. However, whether the depletion of hTERT in these cells can lead to the opposite effects requires further studies. Additional efforts should be made to investigate the universality of our findings with respect to other cancer cell lines.

In conclusion, our findings demonstrate that hTERT overexpression can significantly promote cell migration and invasiveness among human telomerase-positive HepG2 cell lines but does not promote proliferation. Upregulation of metastasis-associated molecules, MMP9 and RhoC, may be one of the most important mechanisms underlying this process. Our study provides experimental evidence of the relationship between telomerase activity and metastatic potential of telomerase-positive tumor cells and also aids in the research into the mechanisms underlying this process. These findings provide a possible explanation of the clinical observation that high telomerase activity predicts poor patient outcome in HCC, particularly in cases of recurrence after $\mathrm{HCC}$ resection.

\section{Acknowledgements}

The authors thank Dr David Ott (NCI-Frederick, USA) and Dr Eugene Barsov for providing the plasmid retroviral expression vector $x \operatorname{lox}(\mathrm{gfp}) \mathrm{TERT}$.

\section{References}

1. Morin GB: The human telomere terminal transferase enzyme is a ribonucleoprotein that synthesizes TTAGGG repeats. Cell 59: 521-529, 1989.

2. Greider CW and Blackburn EH: The telomere terminal transferase of Tetrahymena is a ribonucleoprotein enzyme with two kinds of primer specificity. Cell 51: 887-898, 1987.

3. Greider $\mathrm{CW}$ and Blackburn EH: Identification of a specific telomere terminal transferase activity in Tetrahymena extracts. Cell 43: 405-413, 1985.

4. Feng J, Funk WD, Wang SS, et al: The RNA component of human telomerase. Science 269: 1236-1241, 1995.

5. Toshikuni N, Nouso K, Higashi T, et al: Expression of telomerase-associated protein 1 and telomerase reverse transcriptase in hepatocellular carcinoma. Br J Cancer 82: 833-837, 2000.

6. Nakayama J, Tahara H, Tahara E, et al: Telomerase activation by hTRT in human normal fibroblasts and hepatocellular carcinomas. Nat Genet 18: 65-68, 1998.

7. Ohta K, Kanamaru T, Morita Y, Hayashi Y, Ito H and Yamamoto M: Telomerase activity in hepatocellular carcinoma as a predictor of postoperative recurrence. J Gastroenterol 32: 791-796, 1997.

8. Nakashio R, Kitamoto M, Tahara H, Nakanishi T, Ide T and Kajiyama G: Significance of telomerase activity in the diagnosis of small differentiated hepatocellular carcinoma. Int J Cancer 74: 141-147, 1997.

9. Kojima H, Yokosuka O, Imazeki F, Saisho H and Omata M: Telomerase activity and telomere length in hepatocellular carcinoma and chronic liver disease. Gastroenterology 112: 493-500, 1997.

10. Ohta K, Kanamaru T, Yamamoto M and Saitoh Y: Clinical significance of telomerase activity in hepatocellular carcinoma. Kobe J Med Sci 42: 207-217, 1996.

11. Ide $T$, Tahara H, Nakashio R, Kitamoto M, Nakanishi $T$ and Kajiyama G: Telomerase in hepatocellular carcinogenesis. Hum Cell 9: 283-286, 1996.

12. Taga S, Osaki T, Ohgami A, Imoto H and Yasumoto K: Prognostic impact of telomerase activity in non-small cell lung cancers. Ann Surg 230: 715-720, 1999.

13. Marchetti A, Bertacca G, Buttitta F, et al: Telomerase activity as a prognostic indicator in stage I non-small cell lung cancer. Clin Cancer Res 5: 2077-2081, 1999. 
14. Dome JS, Chung S, Bergemann T, et al: High telomerase reverse transcriptase (hTERT) messenger RNA level correlates with tumor recurrence in patients with favorable histology Wilms tumor. Cancer Res 59: 4301-4307, 1999.

15. Okusa Y, Shinomiya N, Ichikura T and Mochizuki H: Correlation between telomerase activity and DNA ploidy in gastric cancer. Oncology 55: 258-264, 1998.

16. Bechter OE, Eisterer W, Pall G, Hilbe W, Kühr T and Thaler J: Telomere length and telomerase activity predict survival in patients with B cell chronic lymphocytic leukemia. Cancer Res 58: 4918-4922, 1998.

17. Langford LA, Piatyszek MA, Xu R, Schold SC Jr, Wright WE and Shay JW: Telomerase activity in ordinary meningiomas predicts poor outcome. Hum Pathol 28: 416-420, 1997.

18. Hiyama E, Hiyama K, Ohtsu K, et al: Telomerase activity in neuroblastoma: is it a prognostic indicator of clinical behaviour? Eur J Cancer 33: 1932-1936, 1997.

19. Clark GM, Osborne CK, LevittD, Wu F and Kim NW: Telomerase activity and survival of patients with node-positive breast cancer. J Natl Cancer Inst 89: 1874-1881, 1997.

20. Hiyama E, Yokoyama T, Tatsumoto N, et al: Telomerase activity in gastric cancer. Cancer Res 55: 3258-3262, 1995.

21. Hiyama E, Hiyama K, Yokoyama T, Matsuura Y, Piatyszek MA and Shay JW: Correlating telomerase activity levels with human neuroblastoma outcomes. Nat Med 1: 249-255, 1995.

22. Saini N, Srinivasan R, Chawla Y, Sharma S, Chakraborti A and Rajwanshi A: Telomerase activity, telomere length and human telomerase reverse transcriptase expression in hepatocellular carcinoma is independent of hepatitis virus status. Liver Int 29: $1162-1170,2009$.

23. Suda T, Isokawa O, Aoyagi Y, et al: Quantitation of telomerase activity in hepatocellular carcinoma: a possible aid for a prediction of recurrent diseases in the remnant liver. Hepatology 27: 402-406, 1998

24. Tatsuma T, Goto S, Kitano S, Lin YC, Lee CM and Chen CL: Telomerase activity in peripheral blood for diagnosis of hepatoma. J Gastroenterol Hepatol 15: 1064-1070, 2000.

25. Kobayashi T, Kubota K, Takayama $\mathrm{T}$ and Makuuchi $\mathrm{M}$ : Telomerase activity as a predictive marker for recurrence of hepatocellular carcinoma after hepatectomy. Am J Surg 181: 284-288, 2001.

26. Sun B, Huang Q, Liu S, et al: Progressive loss of malignant behavior in telomerase-negative tumorigenic adrenocortical cells and restoration of tumorigenicity by human telomerase reverse transcriptase. Cancer Res 64: 6144-6151, 2004.

27. Brachner A, Sasgary S, Pirker C, et al: Telomerase- and alternative telomere lengthening-independent telomere stabilization in a metastasis-derived human non-small cell lung cancer cell line: effect of ectopic hTERT. Cancer Res 66: 3584-3592, 2006.

28. Yu ST, Chen L, Wang HJ, Tang XD, Fang DC and Yang SM: hTERT promotes the invasion of telomerase-negative tumor cells in vitro. Int J Oncol 35: 329-336, 2009.

29. Yao X, Wang X, Zhang S, Yan L and Zhu H: Inhibitory effect of silencing hTERT gene on growth of human squamous cell carcinoma xenograft in nude mice. Lin Chung Er Bi Yan Hou Tou Jing Wai Ke Za Zhi 25: 939-943, 2011 (In Chinese).

30. George J, Banik NL and Ray SK: Knockdown of hTERT and concurrent treatment with interferon-gamma inhibited proliferation and invasion of human glioblastoma cell lines. Int J Biochem Cell Biol 42: 1164-1173, 2010.

31. Sato N, Maehara N, Mizumoto K, et al: Telomerase activity of cultured human pancreatic carcinoma cell lines correlates with their potential for migration and invasion. Cancer 91: 496-504, 2001.
32. Bagheri S, Nosrati M, Li S, et al: Genes and pathways downstream of telomerase in melanoma metastasis. Proc Natl Acad Sci USA 103: 11306-11311, 2006

33. Nart D, Yaman B, Yilmaz F, Zeytunlu M, Karasu Z and Kiliç M: Expression of matrix metalloproteinase- 9 in predicting prognosis of hepatocellular carcinoma after liver transplantation. Liver Transpl 16: 621-630, 2010.

34. Arii S, Mise M, Harada T, et al: Overexpression of matrix metalloproteinase 9 gene in hepatocellular carcinoma with invasive potential. Hepatology 24: 316-322, 1996.

35. Clark EA, Golub TR, Lander ES and Hynes RO: Genomic analysis of metastasis reveals an essential role for RhoC. Nature 406: 532-535, 2000

36. Ridley AJ: Rho GTPases and cell migration. J Cell Sci 114: 2713-2722, 2001

37. Van Aelst L and D'Souza-Schorey C: Rho GTPases and signaling networks. Genes Dev 11: 2295-2322, 1997.

38. Wang W, Yang LY, Huang GW, et al: Genomic analysis reveals RhoC as a potential marker in hepatocellular carcinoma with poor prognosis. Br J Cancer 90: 2349-2355, 2004.

39. Wang W, Yang LY, Yang ZL, Huang GW and Lu WQ: Expression and significance of RhoC gene in hepatocellular carcinoma. World J Gastroenterol 9: 1950-1953, 2003.

40. Okabe H, Satoh S, Kato T, et al: Genome-wide analysis of gene expression in human hepatocellular carcinomas using cDNA microarray: identification of genes involved in viral carcinogenesis and tumor progression. Cancer Res 61: 2129-2137, 2001.

41. Kleer CG, Griffith KA, Sabel MS, et al: RhoC-GTPase is a novel tissue biomarker associated with biologically aggressive carcinomas of the breast. Breast Cancer Res Treat 93: 101-110, 2005.

42. Shikada Y, Yoshino I, Okamoto T, Fukuyama S, Kameyama T and Maehara Y: Higher expression of RhoC is related to invasiveness in non-small cell lung carcinoma. Clin Cancer Res 9: 5282-5286, 2003.

43. Xue F, Takahara T, Yata Y, et al: Blockade of Rho/Rho-associated coiled coil-forming kinase signaling can prevent progression of hepatocellular carcinoma in matrix metalloproteinase-dependent manner. Hepatol Res 38: 810-817, 2008.

44. Iiizumi M, Bandyopadhyay S, Pai SK, et al: RhoC promotes metastasis via activation of the Pyk2 pathway in prostate cancer. Cancer Res 68: 7613-7620, 2008.

45. Belair CD, Yeager TR, Lopez PM and Reznikoff CA: Telomerase activity: a biomarker of cell proliferation, not malignant transformation. Proc Natl Acad Sci USA 94: 13677-13682, 1997.

46. Smith LL, Coller HA and Roberts JM: Telomerase modulates expression of growth-controlling genes and enhances cell proliferation. Nat Cell Biol 5: 474-479, 2003.

47. Gronthos S, Chen S, Wang CY, Robey PG and Shi S: Telomerase accelerates osteogenesis of bone marrow stromal stem cells by upregulation of CBFA1, osterix, and osteocalcin. J Bone Miner Res 18: 716-722, 2003.

48. Xiang H, Wang J, Mao Y, Liu M, Reddy VN and Li DW: Human telomerase accelerates growth of lens epithelial cells through regulation of the genes mediating RB/E2F pathway. Oncogene 21: 3784-3791, 2002.

49. Bednarek AK, Sahin A, Brenner AJ, Johnston DA and Aldaz CM: Analysis of telomerase activity levels in breast cancer: positive detection at the in situ breast carcinoma stage. Clin Cancer Res 3 : $11-16,1997$. 